\title{
Regionalização para o cultivo do feijão no Rio Grande do Sul com base na interação genótipo $x$ ambiente ${ }^{1}$
}

\author{
Clause Fátima de Brum Piana², João Gilberto Corrêa da Silva³, Irajá Ferreira Antunes ${ }^{4}$
}

\section{RESUMO}

A regionalização de uma cultura agrícola é estratégia utilizada para minimizar efeitos da interação genótipo x ambiente. Esta abordagem baseia-se em resultados de experimentos conduzidos em diversos locais e anos, cuja análise estatística apresenta dificuldades originadas de estrutura de dados incompleta e heterogeneidade da variância do erro experimental. Este estudo teve o objetivo de avaliar a magnitude e a natureza da interação genótipo x ambiente e a possibilidade de estratificação das regiões de cultivo do feijão do Estado do Rio Grande do Sul. Foram considerados dados do Ensaio Estadual de Feijão, conduzido em 24 locais durante oito anos. Foram procedidas análises conjuntas, para cada ano, de dois subconjuntos de quatro anos e do conjunto dos oito anos, e análises de agrupamento, utilizando metodologia que contorna aquelas dificuldades da análise estatística. Os resultados das análises conjuntas anuais revelaram alta significância da interação genótipo x local. Essa interação também foi significativa no conjunto dos oito anos, mas não significativa nos dois subconjuntos de quatro anos. A interação genótipo x local x ano foi altamente significativa nesses três subconjuntos de anos. A análise de agrupamento, baseada nos oito anos, constituiu subregiões incoerentes com as subregiões formadas pelas análises anuais, que também foram inconsistentes entre si. Esses resultados indicaram inadequabilidade da regionalização do Estado do Rio Grande do Sul.

Palavras-chave: Phaseolus vulgaris, componentes de variância, estratificação de ambientes, análise de agrupamento.

\section{ABSTRACT}

\section{Common bean cropping regionalization in Rio Grande do Sul State, Brazil, based on genotype $x$ environment interaction}

The stratification of the crop region is a strategy used to control the effect of the genotype $\mathrm{x}$ environment interaction. This approach is based on results of an experiment conducted in several years and locations, in which the statistical analysis has difficulties originated from the incomplete data structure and variance heterogeneity of the experimental error. This paper reports results of a research on the magnitude and the nature of the genotype x environment interaction and on the convenience for the stratification of the common bean production region in Rio Grande do Sul State, Brazil, for the control of this interaction. Data from the Common Bean Yield State Trial carried out in 24 locations for eight years were evaluated. Joint analyses of each year, of two sub-groups of four and of a set of eight years, as well as cluster analyses, were performed using a methodology that circumvents the statistical difficulties. The results of the annual analyses revealed highly significant genotype x location interaction. This interaction was also significant in the set of the eightyears, but not significant in the two groups of four-years. The genotype $x$ location $x$ year interaction was highly significant in these three sets of years. The cluster analyses based on the eight years constituted sub-regions incoherent with the sub-regions formed by the annual analyses, which were not consistent among themselves. The results point out for the inadequacy of a regional division of the area of common bean production in Rio Grande do Sul.

Key words: Phaseolus vulgaris L., variance components, environment stratification, cluster analysis.

\footnotetext{
Recebido para publicação em 28/10/2010 e aprovado em 10/02/2012

'Parte da tese do doutorado da primeira autora.

${ }^{2}$ Bióloga, Doutora. Universidade Federal de Pelotas, Centro de Desenvolvimento Tecnológico, Campus Porto, Rua Gomes Carneiro, 1, Centro, 96010-610, Caixa Postal 354, 96010900, Pelotas, Rio Grande do Sul, Brasil. clausepiana@yahoo.com.br

${ }^{3}$ Engenheiro-Agrônomo, PhD. Aposentado. Rua Gonçalves Chaves 3063, 96015-560, Pelotas, Rio Grande do Sul, Brasil. jgcs@ufpel.edu.br

${ }^{4}$ Engenheiro-Agrônomo, Doutor. Embrapa Clima Temperado, Rodovia BR 392, Km 78, Campus Universitário, Caixa Postal 103, 96001-970, Pelotas, Rio Grande do Sul, Brasil. iraja@cpact.embrapa.br
} 


\section{INTRODUÇÃO}

No Brasil, o feijão (Phaseolus vulgaris L.) é cultivado numa gama de ambientes ecologicamente distintos. Sua produtividade é baixa e varia muito entre regiões e anos agrícolas (CONAB, 2011), o que revela elevada influência das condições de ambiente. Esse fato torna o feijão uma cultura de risco, o que gera insegurança aos produtores e consequente desestímulo ao seu cultivo.

A alteração dos rendimentos médios relativos de genótipos com a variação do ambiente é um fato comum em pesquisas de melhoramento e significa presença da interação genótipo x ambiente. Essa interação constituise num entrave para a obtenção de genótipos que mantenham rendimentos consistentemente elevados e superiores aos demais, nos locais de uma ampla região. Nessas circunstâncias, dados de apenas rendimentos médios de cultivares não são base adequada para a indicação de cultivares. Um método de melhoramento eficiente deve considerar, também, a estabilidade relativa dos rendimentos entre os locais da região de cultivo (Liang et al., 1966).

Duas estratégias podem ser consideradas para otimizar o rendimento na presença da interação genótipo $\mathrm{x}$ ambiente: (1) indicação de cultivares com adaptabilidade e alta estabilidade de rendimento na região de cultivo e (2) divisão dessa região em subregiões, para as quais possam ser identificados cultivares específicos, bem adaptados e com rendimentos estáveis.

Diante da dificuldade de obtenção de genótipos com estabilidade de rendimento em uma grande região, pesquisadores têm-se voltado para a estratégia da regionalização. A regionalização parte das respostas dos genótipos à gama de ambientes da região de cultivo, considerando os efeitos globais das características do ambiente sobre os genótipos, sem requerer um conhecimento específico dessas características (Cordeiro \& Silva, 1980). Essa abordagem baseia-se em resultados de experimentos conduzidos em diversos locais e anos, supostamente representativos da região. Os métodos de regionalização mais utilizados baseiam-se na análise de agrupamento ou análise de conglomeração ("cluster analysis"). Essa abordagem foi originalmente utilizada por Abou-El-Fittouh et al. (1969) e, desde então, diversos métodos de agrupamento têm sido propostos, com variações quanto ao critério de similaridade, à medida de distância e ao algoritmo de agrupamento que adotam (Mungomery et al., 1974; Byth et al., 1976; Campbell \& Lafever, 1977).

A análise estatística de experimentos regionais de melhoramento genético, conduzidos em diversos locais e anos, suscita diversas dificuldades metodológicas, principalmente as que decorrem de estrutura de dados incompleta e de violação da pressuposição de homogeneidade da variância do erro experimental. Esses problemas, quan- do não são ignorados pelo pesquisador, em geral são tratados de forma inadequada. Silva (1997) fez uma apreciação sobre esses problemas metodológicos com base em uma revisão ampla da literatura.

O modo usual de contornar problemas decorrentes de estrutura fatorial incompleta tem sido a consideração apenas de análises separadas por ano (Cordeiro \& Silva, 1980; Silva, 1982; Murakami \& Cruz, 2004). Entretanto, essa abordagem ignora as interações de local e genótipo com ano e requer a síntese subjetiva dos resultados das análises, separadas dos anos. Por essa razão, ela é questionável em estudos de regionalização. Alguns pesquisadores (Oliveira et al., 2005; Garbuglio et al., 2007; Mendonça et al., 2007) optaram pelo agrupamento de ambientes (combinações de locais e anos). Esse procedimento também não é apropriado, pois dificilmente pode conduzir a resultados interpretáveis e a inferências de importância prática. Essas inferências devem ser aplicáveis a um conjunto de anos no futuro, o que requer sua consistência para os anos considerados na amostra.

Para contornar o problema da heterogeneidade da variância do erro experimental, o procedimento proposto por Cochran (1954) tem sido o mais utilizado. Esse procedimento utiliza o método da análise da variação (método dos momentos), que não considera efeitos aleatórios, apropriadamente, e tenta levar em conta essa heterogeneidade da variância por um ajuste de graus de liberdade pela fórmula de Satterthwaite (1946).

A abordagem apropriada deve fundamentar-se na consideração da estrutura das variâncias e covariâncias dos efeitos aleatórios presentes no modelo estatístico, que leve em conta particularmente a possibilidade de heterogeneidade da variância do erro experimental. Um procedimento para esse propósito é a utilização de métodos baseados na verossimilhança (Searle et al., 1992; Littell et al., 2006). Embora o desenvolvimento dessa metodologia tenha sido iniciado na década de 60 do século XX (Thompson, 1962; Patterson \& Thompson, 1971), ela ainda é pouco utilizada, principalmente porque, sendo muito exigente em recursos computacionais, tornou-se exequível somente após sua recente implementação em programas estatísticos, o que a faz ainda pouco conhecida.

Em feijão, pesquisas relacionadas com a análise de estabilidade do rendimento de grãos e adaptabilidade dos genótipos são muito mais numerosas do que as que visam à estratificação de áreas de cultivo heterogêneas. As pesquisas de Carbonell \& Pompeu (1997) e Oliveira et al. (2005) parecem ser as exceções. Particularmente, no Rio Grande Sul, têm sido realizadas algumas pesquisas com o propósito de identificar genótipos com estabilidade de rendimento e ampla adaptabilidade, mas, até o momento, nenhuma sobre a estratificação da região de cultivo é encontrada na literatura. 
A presente pesquisa explorou dados do Ensaio Estadual de Feijão (EEF) do Rio Grande do Sul, do período de 1987/88 a 1994/95, com dois objetivos principais: avaliar a magnitude e a natureza da interação genótipo x ambiente, utilizando metodologia que leve em conta a heterogeneidade da variância do erro experimental e seja adequada para estrutura de dados incompleta; e identificar possível estratificação da região de cultivo do feijão desse Estado em subregiões dentro das quais genótipos tenham desempenhos relativos estáveis.

\section{MATERIAL E MÉTODOS}

Foram utilizados, nesta pesquisa, dados de rendimento de grãos do Ensaio Estadual de Feijão (EEF), coordenado pela Comissão Estadual de Pesquisa de Feijão (CEPEF) e executado com a colaboração de várias instituições de pesquisa do Estado do Rio Grande do Sul. Esses dados correspondem a um período de oito anos de execução do EEF (1987/88 a 1994/95), em que foram considerados 88 genótipos e 24 locais do Estado. Os locais e os genótipos variaram entre os anos. As combinações de anos e locais constituíram 89 ambientes, apresentados na Tabela 1.

Em todos os ambientes, foi adotado o delineamento em blocos casualizados, com quatro repetições. A parcela compreendeu quatro linhas de $4 \mathrm{~m}$ de comprimento, com espaçamento de $0,5 \mathrm{~m}$ entre linhas e população ideal de 12 plantas por metro linear. Aárea útil da parcela foi constituída dos $3 \mathrm{~m}$ centrais das duas linhas internas, compreendendo $3 \mathrm{~m}^{2}$. A semeadura foi procedida na época da safra, entre o início de setembro e o final de novembro, em todos os anos. A condução dos ensaios seguiu as recomendações técnicas para o cultivo do feijão (IPAGRO, 1986).

Nos anos agrícolas de 1987/88, 1988/89, 1989/90, 1990/ 91, 1991/92, 1992/93, 1993/94 e 1994/95 foram considerados 30, 30, 30, 26, 24, 24, 24 e 28 genótipos, respectivamente. Esses genótipos supostamente representam a população de genótipos de feijão cultivados no Rio Grande do Sul. O requisito importante para essa representatividade é a ampla variação genética, que reflita variações de rendimento de grãos e de outras características morfofisiológicas.

A pesquisa compreendeu as seguintes análises estatísticas dos dados da variável rendimento de grãos: análises conjuntas anuais; análises de dois subconjuntos de quatro anos: de 1987/88 a 1990/91 (anos 1-4) e de 1991/92 a 1994/95 (anos 5-8); análise do conjunto dos oito anos (anos 1-8) e análises de agrupamento de locais para cada ano e para cada um dos subconjuntos de quatro e oito anos. Preliminarmente a essas análises, foram efetuadas as análises individuais dos dados dos 89 ambientes, para a estimação da variância devida ao erro experimental por

Tabela 1. Data da semeadura em cada ambiente (combinação de local e ano) de execução do Ensaio Estadual de Feijão nos anos agrícolas de 1987/88 a 1994/95, no Estado do Rio Grande do Sul

\begin{tabular}{|c|c|c|c|c|c|c|c|c|c|}
\hline \multirow{2}{*}{ Local } & \multicolumn{8}{|c|}{ Ano agrícola } & \multirow{2}{*}{ Total } \\
\hline & $1987 / 88$ & 1988/89 & $1989 / 90$ & $1990 / 91$ & $1991 / 92$ & $1992 / 93$ & $1993 / 94$ & $1994 / 95$ & \\
\hline Canguçu & $19 / 10$ & $22 / 10$ & $30 / 10$ & $26 / 10$ & $22 / 10$ & $07 / 10$ & $22 / 10$ & $07 / 11$ & 8 \\
\hline Iraí & $18 / 09$ & $22 / 09$ & $28 / 09$ & $26 / 09$ & $03 / 10$ & & $11 / 10$ & $23 / 09$ & 7 \\
\hline Maquiné & $25 / 09$ & $19 / 09$ & $21 / 09$ & $06 / 09$ & $10 / 09$ & $21 / 10$ & $08 / 10$ & & 7 \\
\hline Encruzilhada do Sul & & $07 / 10$ & $05 / 10$ & $26 / 09$ & $01 / 10$ & $02 / 10$ & $08 / 10$ & $28 / 10$ & 7 \\
\hline Frederico Westphalen & $21 / 09$ & $29 / 09$ & $27 / 09$ & $11 / 09$ & $02 / 10$ & & & & 5 \\
\hline Erechim & $21 / 10$ & $21 / 10$ & $20 / 10$ & & $22 / 10$ & $20 / 10$ & & & 5 \\
\hline Pelotas & $22 / 10$ & $16 / 10$ & $19 / 10$ & & & $01 / 10$ & $08 / 10$ & & 5 \\
\hline Capão do Leão & & $23 / 10$ & - & & & & $23 / 11$ & $30 / 11$ & 4 \\
\hline Cruz Alta & & $30 / 09$ & & $28 / 09$ & $02 / 10$ & & & - & 4 \\
\hline Santa Maria & & $28 / 09$ & $29 / 09$ & & $12 / 09$ & $30 / 10$ & & & 4 \\
\hline Estrela & & $16 / 09$ & $19 / 09$ & $25 / 09$ & & $01 / 10$ & & & 4 \\
\hline Sarandi & & & & $18 / 10$ & $03 / 10$ & $07 / 10$ & $16 / 10$ & & 4 \\
\hline Veranópolis & & & & & $11 / 10$ & $06 / 10$ & $14 / 10$ & - & 4 \\
\hline Passo Fundo & & & & & $05 / 10$ & $01 / 10$ & $07 / 10$ & - & 4 \\
\hline Sobradinho & $06 / 10$ & & & $18 / 10$ & $01 / 10$ & & & & 3 \\
\hline Caiçara & & & & & $03 / 10$ & & $08 / 10$ & $21 / 09$ & 3 \\
\hline Santo Augusto & & & & & $27 / 09$ & $15 / 10$ & $26 / 10$ & & 3 \\
\hline Júlio de Castilhos & & & & 28/09 & $21 / 10$ & & & & 2 \\
\hline Santa Rosa & & $22 / 09$ & & & & & & & 1 \\
\hline Coronel Bicaco & & $29 / 09$ & & & & & & & 1 \\
\hline Arroio do Tigre & & & & $29 / 09$ & & & & & 1 \\
\hline Nonoai & & & & & $16 / 10$ & & & & 1 \\
\hline Rio Grande & & & & & & $02 / 10$ & & & 1 \\
\hline Augusto Pestana & & & & & & & & - & 1 \\
\hline Total & 7 & 13 & 10 & 11 & 16 & 12 & 11 & 9 & 89 \\
\hline
\end{tabular}

Traço significa dado não disponível. 
ambiente, e o teste $\chi^{2}$ de Bartlett (1937) da homogeneidade dessa variância para cada ano e para os anos 1-4, 5-8 e 18. Os resultados revelaram heterogeneidade da variância do erro experimental altamente significativa $(\mathrm{P}<0,0001)$ para todos esses subconjuntos de ambientes.

As análises anuais consideraram os conjuntos completos de locais e genótipos presentes em cada ano. Entretanto, as análises conjuntas de anos compreenderam subconjuntos selecionados de locais e de genótipos. A escolha dos locais e dos genótipos, para cada uma destas análises, foi procedida pelo exame tentativo das combinações de genótipos e locais, visando à redução do desbalanceamento da estrutura dos dados, suficiente para a predição dos efeitos da interação genótipo x local, com o menor prejuízo possível para a representação dos ambientes de cultivo do feijão e da população de genótipos. Os locais considerados nas três análises conjuntas são identificados na Tabela 2. Essas análises compreenderam: 36 ambientes e 17 genótipos para os anos 1-4, 41 ambientes e 16 genótipos para os anos 5-8, e 72 ambientes e dez genótipos para os oito anos.

Em razão de se buscar uma regionalização de longo prazo, geral para os locais de cultivo do Rio Grande do Sul e para a coleção de cultivares de feijão produzidos nesse Estado, os fatores ano, local e genótipo foram considerados aleatórios.

As análises conjuntas foram procedidas de acordo com os seguintes modelos estatísticos com base nas observações por parcela:

1) Análises anuais: $y_{i j k}=\mu+l_{j}+b_{k(j)}+g_{i}+g l_{i j}+e_{i j k}$, $\mathrm{i}=1,2, \ldots, \mathrm{g} ; \mathrm{j}=1,2, \ldots, \mathrm{h} ; \mathrm{k}=1,2, \ldots, \mathrm{b}$, em que: $\mu$ : rendimento médio esperado; $1_{\mathrm{j}}$ : efeito aleatório do local $\mathrm{j} ; \mathrm{b}_{\mathrm{k}(\mathrm{j})}$ : efeito aleatório do bloco $\mathrm{k}$ do local j; $\mathrm{g}_{\mathrm{i}}$ : efeito aleatório do

Tabela 2. Frequências dos locais considerados nas análises conjuntas de quatro e dos oito anos, no Estado do Rio Grande do Sul

\begin{tabular}{lccc}
\hline & \multicolumn{3}{c}{ Conjunto de anos } \\
\cline { 2 - 4 } Local & $\mathbf{1 9 8 7 / 8 8}$ a & $\mathbf{1 9 9 1 / 9 2}$ a & $\mathbf{1 9 8 7 / 8 8}$ a \\
& $\mathbf{1 9 9 0 / 9 1}$ & $\mathbf{1 9 9 4 / 9 5}$ & $\mathbf{1 9 9 4 / 9 5}$ \\
\hline Canguçu & 4 & 4 & 8 \\
Iraí & 3 & 3 & 7 \\
Encruzilhada do Sul & 3 & 4 & 7 \\
Maquiné & 4 & 3 & 7 \\
Frederico Westphalen & 4 & - & 5 \\
Erechim & 3 & 2 & 5 \\
Pelotas & 3 & 2 & 5 \\
Capão do Leão & 2 & 2 & 4 \\
Cruz Alta & 2 & 2 & 4 \\
Santa Maria & 2 & 2 & 4 \\
Estrela & 3 & - & 4 \\
Sarandi & - & 3 & 4 \\
Veranópolis & - & 4 & 4 \\
Passo Fundo & - & 4 & 4 \\
Caiçara & - & 3 & - \\
Santo Augusto & - & 3 & - \\
Sobradinho & 2 & - & - \\
\hline
\end{tabular}

Traço indica local desconsiderado no respectivo conjunto de anos. genótipo i; gl $\mathrm{gl}_{\mathrm{ij}}$ : efeito aleatório da interação do genótipo i com o local $\mathrm{j} ; \mathrm{e}_{\mathrm{ijk}}$ : erro experimental aleatório.

2) Análises de conjuntos de anos: $y_{\mathrm{ijks}}=\mu+\mathrm{a}_{\mathrm{s}}+\mathrm{l}_{\mathrm{j}}+\mathrm{la}_{\mathrm{js}}+$ $\mathrm{b}_{\mathrm{k}(\mathrm{js})}+\mathrm{g}_{\mathrm{i}}+\mathrm{ga}_{\mathrm{is}}+\mathrm{gl}_{\mathrm{ij}}+\mathrm{gla}_{\mathrm{ijs}}+\mathrm{e}_{\mathrm{ijks}}, \mathrm{i}=1,2, \ldots, \mathrm{g} ; \mathrm{j}=1,2, \ldots, \mathrm{h}$; $\mathrm{k}=1,2, \ldots, \mathrm{b} ; \mathrm{s}=1,2, \ldots, \mathrm{a} ;$ em que: $\mathrm{a}$ : efeito aleatório do ano $\mathrm{s} ; \mathrm{la}_{\mathrm{js}}$ : efeito aleatório da interação do local j com o ano s; $\mathrm{b}_{\mathrm{k}(\mathrm{js})}$ : efeito aleatório do bloco $\mathrm{k}$ do local $\mathrm{j}$ e ano s; $\mathrm{ga}_{\mathrm{is}}$ : efeito aleatório da interação do genótipo i com o ano s; gla $_{\mathrm{ijs}}$ : efeito aleatório da interação do genótipo i com o local j e o ano s; $\mathrm{e}_{\mathrm{ijks}}$ : erro experimental aleatório.

Para os efeitos aleatórios de bloco, genótipo, local e ano e as interações duplas e triplas destes três últimos efeitos, foram admitidas as pressuposições de distribuição normal e independência dos efeitos de uma mesma observação e de observações distintas. Os correspondentes componentes de variância foram denotados por $\sigma_{\mathrm{B}}^{2}, \sigma_{\mathrm{G}}^{2}, \sigma_{\mathrm{L}}^{2}, \sigma_{\mathrm{A}}^{2}, \sigma_{\mathrm{GL}}^{2}, \sigma_{\mathrm{GA}}^{2}$ e $\sigma_{\mathrm{GLA}}^{2}$.

Esses modelos estatísticos são formulações particulares do modelo linear misto geral, descrito por Searle et al. (1992) e Littell et al. (2006), cuja equação é expressa na seguinte forma matricial:

$$
\mathbf{y}=\mathbf{X b}+\mathbf{Z u}+\mathbf{e},
$$

em que: y é o vetor dos valores da variável resposta, registrados nas $n$ unidades de observação, $\mathbf{X}$ é uma matriz conhecida $\mathrm{n} \times \mathrm{p}$ de posto igual ou inferior a $\mathrm{p}$, b é um vetor $\mathrm{p} \times 1$ de efeitos fixos desconhecidos (parâmetros), $\mathbf{Z}$ é uma matriz $\mathrm{m} \times \mathrm{q}$, também conhecida, $\mathbf{u}$ é um vetor $\mathrm{q} \times 1 \mathrm{de}$ efeitos aleatórios e e é um vetor aleatório $\mathrm{n} \times 1$. O termo $\mathbf{X b}$ é a parte da equação que expressa os efeitos fixos e $\mathbf{Z u}$ é a parte que expressa os efeitos aleatórios, excluídos os erros, que compõem o vetor e. Na presente aplicação, o vetor $\mathbf{b}$ compreende apenas a média geral esperada $\mu$, os elementos do vetor u são os efeitos de bloco, genótipo, local e ano e das interações de genótipo, local e ano, cuja presença depende do modelo, e os elementos do vetor $\mathbf{e}$ são os efeitos do erro experimental. Os elementos das matrizes $\mathbf{X}$ e $\mathbf{Z}$ são valores de variáveis indicadoras que expressam a estrutura do experimento: $\mathbf{X}$ é um vetor $\mathrm{nx} 1$ cujos elementos são todos iguais a 1 e $\mathbf{Z}$ é a matriz de incidência dos efeitos aleatórios presentes no modelo estatístico considerado na análise conjunta particular. A variância do vetor dos valores observados da variável resposta é: $\mathbf{V}=\mathbf{Z G} \mathbf{Z}^{\mathrm{T}}+\mathbf{R}$, em que $\mathbf{G}=\operatorname{Var}(\mathbf{u})$ e $\mathbf{R}=\operatorname{Var}(\mathbf{e})$.

Esse modelo linear misto geral permite flexibilidade para a modelagem da matriz de covariância das observações $\mathbf{V}$, pelas especificações apropriadas das matrizes $\mathbf{G}$ e R. Essa facilidade é especialmente interessante, por possibilitar a modelagem da matriz de covariância dos erros $\mathbf{R}$ de modo a levar em conta a heterogeneidade da variância dos erros experimentais entre ambientes. Em geral, as inferências de interesse são: estimação e testes de hipóteses referentes ao vetor de efeitos fixos $\mathbf{b}$, predição dos efeitos aleatórios do vetor $\mathbf{u}$ e estimação e testes de hipóteses referentes aos componentes de variância correspondentes a esses efeitos aleatórios, ou seja, aos elementos das matrizes $\mathbf{G}$ e R. A 
abordagem para essas inferências, reconhecida como mais apropriada, é baseada na verossimilhança. Uma descrição resumida dos procedimentos de inferência para esses modelos é apresentada por Littell et al. (2006). Descrição mais detalhada pode ser encontrada em Searle et al. (1992).

Nesta pesquisa, são de interesse as predições dos efeitos aleatórios da interação de genótipo e local e as estimações e os testes das hipóteses de nulidade dos componentes de variância referentes aos efeitos de genótipo, local e ano e de suas interações. Foi utilizado o método da máxima verossimilhança restrita e os testes de Wald e da razão de verossimilhança. Para o processamento das análises para essas inferências, foi utilizado o procedimento MIXED do "Statistical Analysis System" (SAS Institute, 2008). O PROC MIXED propiciou a flexibilidade necessária para a especificação apropriada das estruturas de covariâncias induzidas pelos modelos estatísticos adotados.

As análises de agrupamento para regionalização foram procedidas pelo método proposto por Sokal \& Michener (1958) e descrito por Cordeiro \& Silva (1980), que efetua aglomeração sequencial, hierárquica e sem superposição.

Nas análises de agrupamento anuais e para conjuntos de anos, cada local foi representado pelo vetor dos valores preditos da interação genótipo x local, providos pelas respectivas análises separadas e conjuntas de anos. A similaridade de dois locais j e j' foi medida pela distância euclidiana, definida como a distância média entre os locais em um espaço de g dimensões, cuja expressão é:

$$
\mathrm{d}_{\mathrm{jj} j^{\prime}}=\left\{\left[\sum_{\mathrm{i}=1}^{\mathrm{g}}\left(\widehat{\mathrm{gl}}_{\mathrm{ij}}-\widehat{\mathrm{gl}}_{\mathrm{ij}}\right)^{2}\right] \frac{1}{\mathrm{~g}}\right\}^{1 / 2},
$$

em que: $\widehat{g l}_{i j}$ : valor predito da interação entre o genótipo i e o local j; $\widehat{g l}_{\mathrm{ij}}$ : valor predito da interação entre o genótipo i e o local j'; g: número de genótipos comuns aos locais j e j'.

O método de Sokal \& Michener (1958) opera sobre a matriz de similaridades e agrupa em seu primeiro estágio os dois locais mais similares. Nos estágios subsequentes, a distância entre duas entidades (locais ou grupos de locais) é determinada pela estratégia combinatória de Lance e Williams (Sneath \& Sokal, 1973), que utiliza como medida de distância: $d_{\left(j, j^{\prime}\right) j^{\prime \prime}}=\frac{n_{j}}{n_{j, j^{\prime}}} d_{j, j^{\prime \prime}}+\frac{n_{j^{\prime}}}{n_{j, j^{\prime}}} d_{j^{\prime}, j^{\prime \prime}}$, em

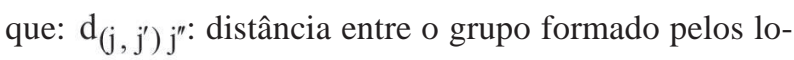

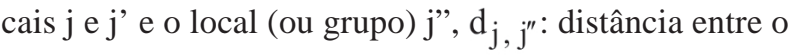
local $\mathrm{j}$ e o local (ou grupo) $\mathrm{j}$ ”, $\mathrm{n}_{\mathrm{j}}$ : número de locais no grupo $\mathrm{j}$ (se $\mathrm{n}_{\mathrm{j}}=1$, então grupo=local), $\mathrm{n}_{\mathrm{j}} \mathrm{j}^{\prime}$ : número de locais no grupo formado pelos locais $\mathrm{j}$ e j'. O processo continua até que todos os locais estejam em um único grupo. Os resultados dessas análises são apresentados em dendrogramas, utilizando-se a distância euclidiana como escala. As subregiões são formadas pelos grupos de locais em um mesmo ramo, identificados por uma seção transversal traçada pelo ponto de parada do processo de agrupamento. Foi adotada como critério para o ponto de parada a não significância do componente da interação genótipo x local dentro de regiões. Para determinar esse ponto de cada processo de agrupamento, efetuou-se a correspondente análise separada ou conjunta de anos para cada estágio do processo, com a inclusão no modelo estatístico do efeito do fator subregião, considerado como fixo, e dos efeitos decorrentes da consideração desse fator adicional. Em cada uma dessas análises, foi efetuado o teste da razão de verossimilhança, para verificar a significância do componente de variância correspondente à interação genótipo x local dentro de regiões.

\section{RESULTADOS E DISCUSSÃO}

Os resultados das análises conjuntas dos locais para cada ano (Tabela 3) mostraram que os componentes de variância referentes a genótipo, local e interação genótipo $\mathrm{x}$ local foram significativos para todos os anos, exceto o componente local no ano de 1987/88. A significância da variância da interação genótipo x local indica que o comportamento relativo dos genótipos altera-se entre locais dentro do mesmo ano.

Na Tabela 4, são apresentados os resultados das análises conjuntas de quatro e dos oito anos. Observa-se que, para os dois conjuntos de quatro anos, as interações genótipo $\mathrm{x}$ local $\mathrm{x}$ ano e local $\mathrm{x}$ ano foram altamente significativas, mas as interações genótipo $\mathrm{x}$ ano e genótipo $\mathrm{x}$ local foram não significativas. Os resultados para o conjunto dos oito anos foram similares, com exceção da significância da interação genótipo x local.

A significância manifestada pela interação genótipo x local em todas as análises anuais indica que o desempenho relativo dos genótipos de feijão, em um ano específico, varia entre locais. Entretanto, resultados de análises conjuntas anuais não devem ser interpretados isoladamente, pois não levam em conta a interação genótipo $\mathrm{x}$ local x ano. A significância desta interação nas três análises conjuntas de anos (Tabela 4) evidencia que a variação do comportamento relativo dos genótipos entre locais não é consistente entre anos. De fato, os resultados das análises dos anos 1-4 e 5-8 mostraram ausência de significância da interação genótipo x local, o que indica que os rendimentos relativos dos genótipos médios para quatro anos foram homogêneos entre locais. Entretanto, a significância dessa interação no período maior de oito anos sugere a possibilidade de não consistência do comportamento relativo dos genótipos entre locais, ao longo 
dos anos. Esse fato justificou uma tentativa de estratificação da região de cultivo do feijão do Estado do Rio Grande do Sul. Ademais, a amplitude desta região e a diversificação de suas características ecológicas e tecnológicas sugerem a possibilidade de que uma regionalização contribua para acelerar o processo de ganho genético nessa área geográfica.
Os resultados dos agrupamentos de locais procedidos, separadamente, para cada um dos oito anos, são apresentados nos dendrogramas da Figura 1 e sumarizados na Tabela 5. Os pontos de parada do processo de agrupamento são representados nos dendrogramas por segmentos de retas verticais tracejados. Os principais resultados observados nessas análises foram: alta frequência de

Tabela 3. Estimativas e resultados dos testes de significâncias dos componentes de variância para os anos individuais, no Estado do Rio Grande do Sul

\begin{tabular}{|c|c|c|c|c|}
\hline \multirow{2}{*}{ Ano } & \multirow{2}{*}{$\begin{array}{l}\text { Componente de } \\
\text { variância }^{1}\end{array}$} & \multirow{2}{*}{ Estimativa } & \multicolumn{2}{|c|}{ Teste de Wald } \\
\hline & & & $\mathbf{Z}$ & Prob. $>\mathrm{Z}$ \\
\hline \multirow{3}{*}{$1987 / 88$} & $\sigma_{G}^{2}$ & 19.327 & 2,96 & 0,0015 \\
\hline & $\sigma_{L}^{2}$ & 284.152 & 1,64 & 0,0505 \\
\hline & $\sigma_{\mathrm{GL}}^{2}$ & 11.594 & 2,50 & 0,0062 \\
\hline \multirow{3}{*}{$1988 / 89$} & $\sigma_{G}^{2}$ & 26.457 & 3,41 & 0,0003 \\
\hline & $\sigma_{L}^{2}$ & 444.410 & 2,39 & 0,0084 \\
\hline & $\sigma_{\mathrm{GL}}^{2}$ & 17.149 & 5,69 & $<0,0001$ \\
\hline \multirow{3}{*}{$1989 / 90$} & $\sigma_{\mathrm{G}}^{2}$ & 12.844 & 2,85 & 0,0022 \\
\hline & $\sigma_{L}^{2}$ & 415.996 & 2,08 & 0,0190 \\
\hline & $\sigma_{\mathrm{GL}}^{2}$ & 21.533 & 4,14 & $<0,0001$ \\
\hline \multirow{3}{*}{ 1990/91 } & $\sigma_{G}^{2}$ & 17.192 & 2,95 & 0,0016 \\
\hline & $\sigma_{\mathrm{L}}^{2}$ & 274.064 & 2,17 & 0,0149 \\
\hline & $\sigma_{\mathrm{GL}}^{2}$ & 13.379 & 3,80 & $<0,0001$ \\
\hline \multirow{3}{*}{$1991 / 92$} & $\sigma_{G}^{2}$ & 25.869 & 2,79 & 0,0026 \\
\hline & $\sigma_{L}^{2}$ & 338.629 & 2,67 & 0,0038 \\
\hline & $\sigma_{\mathrm{GL}}^{2}$ & 49.677 & 6,76 & $<0,0001$ \\
\hline \multirow{3}{*}{$1992 / 93$} & $\sigma_{G}^{2}$ & 21.794 & 2,50 & 0,0063 \\
\hline & $\sigma^{{ }^{G}}$ & 307.224 & 2,29 & 0,0112 \\
\hline & $\sigma_{G L}^{2}$ & 19.315 & 5,44 & $<0,0001$ \\
\hline \multirow{3}{*}{ 1993/94 } & $\sigma_{G}^{2}$ & 26.008 & 2,83 & 0,0023 \\
\hline & $\sigma_{L}^{2}$ & 295.802 & 2,14 & 0,0160 \\
\hline & $\sigma_{\mathrm{GL}}^{2}$ & 21.475 & 3,95 & $<0,0001$ \\
\hline \multirow{3}{*}{$1994 / 95$} & $\sigma_{\mathrm{G}}^{2}$ & 10.335 & 2,28 & 0,0114 \\
\hline & $\sigma^{2}$ & 436.892 & 1,95 & 0,0257 \\
\hline & $\sigma_{G L}^{2}$ & 30.927 & 5,24 & $<0,0001$ \\
\hline
\end{tabular}

${ }^{1} \sigma_{\mathrm{G}}^{2}$ :variância de genótipo, $\sigma_{\mathrm{L}}^{2}$ :variância de local, $\sigma_{\mathrm{GL}}^{2}$ :variância da interação genótipo x local.

Tabela 4. Estimativas e resultados dos testes de significâncias dos componentes de variância para os subconjuntos de quatro e o conjunto de oito anos, no Estado do Rio Grande do Sul

\begin{tabular}{|c|c|c|c|c|c|c|}
\hline \multirow{3}{*}{$\begin{array}{l}\text { Componente de } \\
\text { variância }^{1}\end{array}$} & \multicolumn{6}{|c|}{ Conjunto de anos } \\
\hline & \multicolumn{2}{|c|}{ 1987/88 a 1990/91 } & \multicolumn{2}{|c|}{ 1991/92 a 1994/95 } & \multicolumn{2}{|c|}{$1987 / 88$ a $1994 / 95$} \\
\hline & Estimativa & Prob. $>\mathrm{Z}$ & Estimativa & Prob. $>\mathrm{Z}$ & Estimativa & Prob. $>\mathrm{Z}$ \\
\hline$\sigma_{\mathrm{A}}^{2}$ & 0 & - & 50.320 & 0,2156 & 33.100 & 0,1585 \\
\hline$\sigma_{L}^{2}$ & 227.890 & 0,0376 & 114.474 & 0,0659 & 140.796 & 0,0356 \\
\hline$\sigma_{G}^{2}$ & 26.469 & 0,0040 & 25.895 & 0,0058 & 29.071 & 0,0209 \\
\hline$\sigma_{\mathrm{LA}}^{2}$ & 182.070 & 0,0006 & 211.293 & 0,0004 & 224.576 & $<0,0001$ \\
\hline$\sigma_{G A}^{2}$ & 131 & 0,4562 & 0 & - & 0 & - \\
\hline$\sigma_{\mathrm{GL}}^{2}$ & 3.883 & 0,0793 & 3.693 & 0,1323 & 4.386 & 0,0470 \\
\hline$\sigma_{\mathrm{GLA}}^{2}$ & 19.556 & $<0,0001$ & 33.229 & $<0,0001$ & 25.667 & $<0,0001$ \\
\hline
\end{tabular}

${ }^{1} \sigma_{\mathrm{A}}^{2}$ : variância de ano, $\sigma_{\mathrm{L}}^{2}$ : variância de local, $\sigma_{\mathrm{G}}^{2}$ : variância de genótipo, $\sigma_{\mathrm{LA}}^{2}$ : variância da interação local x ano, $\sigma_{\mathrm{GA}}^{2}$ : variância da interação genótipo x ano, $\sigma_{\mathrm{GL}}^{2}$ : variância da interação genótipo x local, $\sigma_{\mathrm{GLA}}^{2}$ : variância da interação genótipo x local x ano.

Rev. Ceres, Viçosa, v. 59, n.2, p. 213-224, mar/abr, 2012 

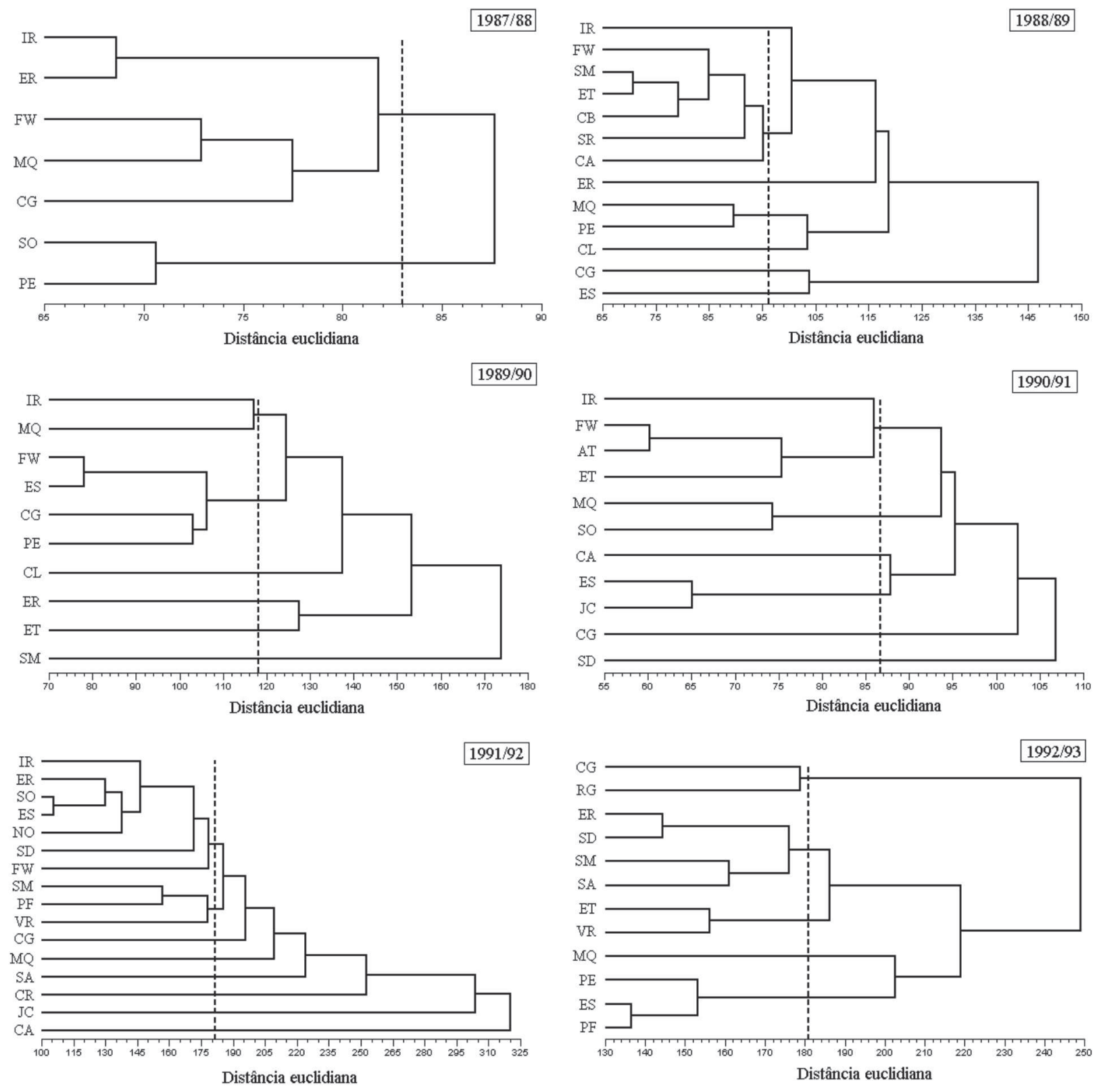

$1993 / 94$

$1994 / 95$
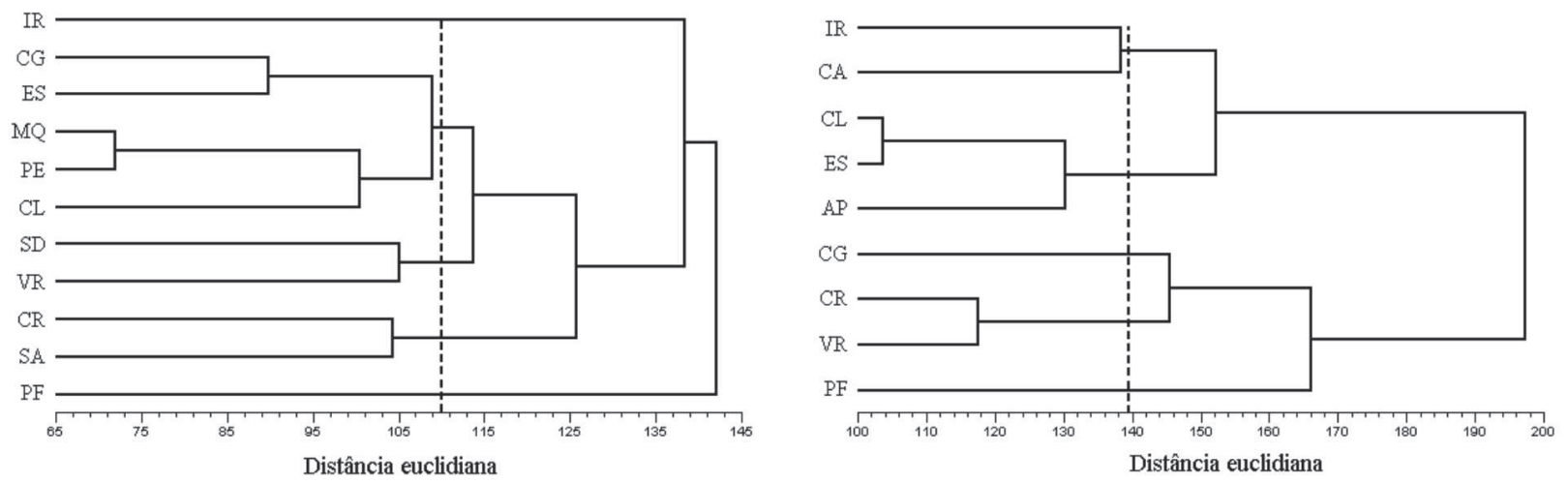

Figura 1. Dendrograma resultante do agrupamento dos locais, para cada um dos oito anos, no Estado do Rio Grande do Sul. IR - Iraí; FW - Frederico Westphalen; CG - Canguçu; ER - Erechim; MQ - Maquiné; SO - Sobradinho; PE - Pelotas; CL - Capão do Leão; CA - Cruz Alta; SM - Santa Maria; SR - Santa Rosa; ES - Encruzilhada do Sul; ET - Estrela; CB - Coronel Bicaco; AT - Arroio do Tigre; JC - Júlio de Castilhos; SD - Sarandi; NO - Nonoai; CR - Caiçara; VR - Veranópolis; PF - Passo Fundo; SA - Santo Augusto; RG - Rio Grande; AP - Augusto Pestana. 
subregiões formadas por apenas um ou dois locais, inconsistência das subregiões formadas entre os anos e ausência de relação entre os agrupamentos de locais e suas posições geográficas. Ademais, apenas três subconjuntos de locais apareceram agrupados, com maior frequência, do que separados, e há um único caso em que os locais só ocorreram agrupados.

Em todos os três conjuntos de anos considerados, a interação local x ano foi o componente de variância de estimativa mais elevada e, consideravelmente, mais signi-

Tabela 5. Subregiões resultantes das análises de agrupamento anuais, no Estado do Rio Grande do Sul

\begin{tabular}{|c|c|c|c|}
\hline Ano & $\begin{array}{l}\text { Número } \\
\text { de locais }\end{array}$ & $\begin{array}{l}\text { Número de } \\
\text { subregiões }\end{array}$ & Subregiões \\
\hline $1987 / 88$ & 7 & 2 & $\begin{array}{l}\text { I: Frederico Westphalen, Maquiné, Canguçu, Iraí e Erechim } \\
\text { II: Sobradinho e Pelotas }\end{array}$ \\
\hline $1988 / 89$ & 13 & 7 & $\begin{array}{l}\text { I: Frederico Westphalen, Cruz Alta, Santa Maria, Santa Rosa, Estrela e Coronel Bicaco } \\
\text { II: Maquiné e Pelotas } \\
\text { III: Iraí } \\
\text { IV: Canguçu } \\
\text { V: Capão do Leão } \\
\text { VI: Encruzilhada do Sul } \\
\text { VII: Erechim }\end{array}$ \\
\hline $1989 / 90$ & 10 & 6 & $\begin{array}{l}\text { I: Frederico Westphalen, Canguçu, Pelotas e Encruzilhada do Sul } \\
\text { II: Iraí e Maquiné } \\
\text { III: Erechim } \\
\text { IV: Capão do Leão } \\
\text { V: Santa Maria } \\
\text { VI: Estrela }\end{array}$ \\
\hline 1990/91 & 11 & 6 & $\begin{array}{l}\text { I: Iraí, Frederico Westphalen, Estrela e Arroio do Tigre } \\
\text { II: Encruzilhada do Sul e Júlio de Castilhos } \\
\text { III: Maquiné e Sobradinho } \\
\text { IV: Cruz Alta } \\
\text { V: Canguçu } \\
\text { VI: Sarandi }\end{array}$ \\
\hline $1991 / 92$ & 16 & 8 & $\begin{array}{l}\text { I: Irá́, Frederico Westphalen, Erechim, Encruzilhada do Sul, Sarandi, Sobradinho e Nonoai } \\
\text { II: Santa Maria, Veranópolis e Passo Fundo } \\
\text { III: Canguçu } \\
\text { IV: Maquiné } \\
\text { V: Cruz Alta } \\
\text { VI: Júlio de Castilhos } \\
\text { VII: Santo Augusto } \\
\text { VIII: Caiçara }\end{array}$ \\
\hline $1992 / 93$ & 12 & 5 & $\begin{array}{l}\text { I: Erechim, Santa Maria, Sarandi e Santo Augusto } \\
\text { II: Pelotas, Encruzilhada do Sul e Passo Fundo } \\
\text { III: Canguçu e Rio Grande } \\
\text { IV: Estrela e Veranópolis } \\
\text { V: Maquiné }\end{array}$ \\
\hline $1993 / 94$ & 11 & 5 & $\begin{array}{l}\text { I: Canguçu, Maquiné, Capão do Leão, Encruzilhada do Sul e Pelotas } \\
\text { II: Sarandi e Veranópolis } \\
\text { III: Caiçara e Santo Augusto } \\
\text { IV: Iraí } \\
\text { V: Passo Fundo }\end{array}$ \\
\hline $1994 / 95$ & 9 & 5 & $\begin{array}{l}\text { I: Capão do Leão, Encruzilhada do Sul e Augusto Pestana } \\
\text { II: Caiçara e Veranópolis } \\
\text { III: Iraí e Cruz Alta } \\
\text { IV: Canguçu } \\
\text { V: Passo Fundo }\end{array}$ \\
\hline
\end{tabular}

Rev. Ceres, Viçosa, v. 59, n.2, p. 213-224, mar/abr, 2012 
ficativo, exceto pela interação tripla genótipo x local x ano. Isso significa que a variação de características do ambiente, com o tempo, pode favorecer um local num determinado ano e desfavorecê-lo em outro. Esse fato pode ser uma explicação para a heterogeneidade dos agrupamentos anuais. Alguma incoerência desses agrupamentos também pode ter decorrido da alteração dos conjuntos de locais e de genótipos, de um ano para o outro. É provável que a variação dos locais tenha maior importância para a explicação dessa inconsistência do que a dos genótipos, dado que em todos os anos os números de locais considerados nessas análises (que variaram de 7 a 16) foram menores do que os de genótipos (de 24 a 30).

A homogeneidade dos rendimentos relativos dos genótipos, entre locais, revelada pelas análises conjuntas dos anos 1-4 e 5-8, dispensou as análises de agrupamento de locais para esses subconjuntos de anos. A análise de agrupamento conjunta dos oito anos foi considerada a mais importante, pois a maior repetição de anos possibilitou melhor representatividade das características do ambiente, que se manifestam de forma diferenciada de um ano para outro, como as ocorrências de doenças e pragas, a distribuição de chuvas e as mudanças de temperatura. Essa análise compreendeu somente os 14 locais que permaneceram no EEF por, no mínimo, quatro anos. $\mathrm{O}$ resultado desse processo de agrupamento é representado no dendrograma da Figura 2 e, as subregiões, constituídas pelo agrupamento dos locais, são apresentadas no mapa da Figura 3.

O agrupamento desses 14 locais resultou na formação de seis subregiões, as quais apresentam as seguintes composições: Subregião I: Iraí, Encruzilhada do Sul, Cruz Alta e Santa Maria; Subregião II: Frederico Westphalen e Erechim; Subregião III: Maquiné, Capão do Leão e Pelotas;
Subregião IV: Estrela, Veranópolis e Sarandi; Subregião V: Passo Fundo; Subregião VI: Canguçu. Conforme pode ser observado na Figura 3, os locais que constituem essas subregiões, na maioria dos casos, não apresentam proximidade geográfica. Contudo, algumas subregiões guardam semelhança em relação a características edafoclimáticas de seus componentes (Tabela 6). Os dois locais que compõem a Subregião II são semelhantes, quanto a características de solo e clima, e apresentam relativa proximidade geográfica. As Subregiões I e IV, por outro lado, destacam-se pela heterogeneidade de seus componentes. Dois dos três locais que compõem a Subregião III assemelham-se em relação à altitude e ao clima.

Ainda que não seja possível uma identificação clara de subregiões para o conjunto dos oito anos, a possibilidade de regionalização não deve ser descartada. Entretanto, a utilidade prática da regionalização, indicada pela análise conjunta dos oito anos, depende de sua consistência com os agrupamentos formados pelas análises anuais e dos dois conjuntos de quatro anos. A análise de agrupamento, para o conjunto dos oito anos, constituiu subregiões incoerentes com as subregiões formadas pelas análises anuais que, por sua vez, foram inconsistentes entre si. Essa falta de coerência estende-se para os dois conjuntos de quatro anos, para os quais correspondeu a formação de um único grupo de locais.

A heterogeneidade dos agrupamentos anuais e de subconjuntos de anos é consequência da elevada interação genótipo x local x ano e sua preponderância sobre a interação genótipo x local. Por outro lado, a inexistência de relação das subregiões constituídas com as disposições geográficas dos locais decorre de o comportamento relativo dos genótipos entre locais não depender de suas distâncias geográficas.

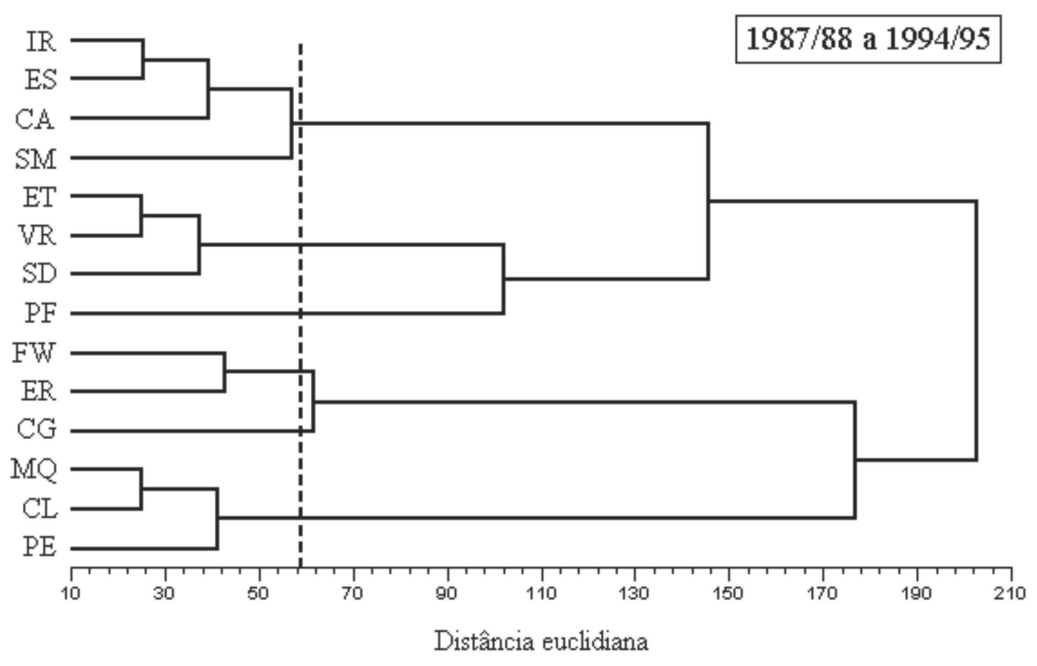

Figura 2. Dendrograma resultante do agrupamento dos locais no período dos oito anos (1987/88 a 1994/95), no Estado do Rio Grande do Sul. IR - Iraí; FW - Frederico Westphalen; CG - Canguçu; ER - Erechim; MQ - Maquiné; PE - Pelotas; CL - Capão do Leão; CA - Cruz Alta; SM - Santa Maria; ES - Encruzilhada do Sul; ET - Estrela; SD - Sarandi; VR - Veranópolis; PF - Passo Fundo. 
Esses fatos evidenciam a inadequabilidade da proposição de uma regionalização de longo prazo do Estado do Rio Grande do Sul, para controle da interação genótipo x local com base no conjunto de dados utilizados nesta pesquisa.

É importante observar, entretanto, que essas inferências podem ter sido afetadas por falhas das técni- cas experimentais adotadas na condução do EEF, nos anos de 1987/88 a 1994/95, detectadas durante a execução desta pesquisa, particularmente a falta de uniformidade dessas técnicas entre os ambientes. Essa desuniformidade é manifestada pelas elevadas variações da data de semeadura entre ambientes e do estande por parcela. De fato, a Tabela 1 mostra que a data de semeadura teve uma varia-

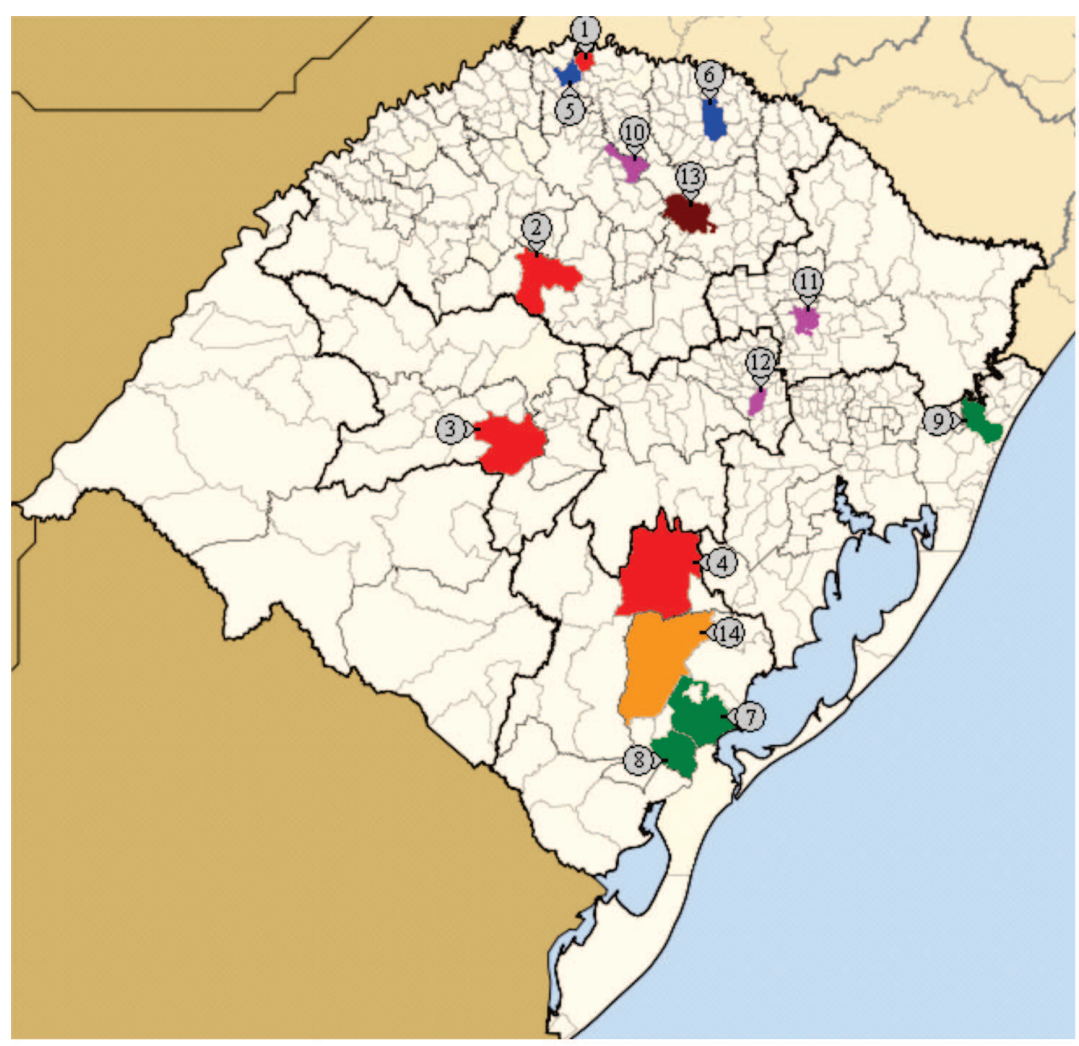

Subregião I:

1-Iraí

2-Cruz Alta

3-Santa Maria

4-Encruzilhada do Sul

Subregião II:

5-Frederico Westphalen

6-Erechim

\section{Subregião III:}

7-Pelotas

8-Capão do Leão

9-Maquiné

\section{Subregião IV:}

10-Sarandi

11-Estrela

12-Veranópolis

\section{Subregião V:}

13-Passo Fundo

\section{Subregião VI:}

14-Canguçu

Figura 3. Subregiões resultantes do agrupamento de locais no período de 1987/88 a 1994/95, no Estado do Rio Grande do Sul.

Tabela 6. Características geográficas, edáficas e climáticas dos 14 locais que compuseram as seis subregiões no período de 1987/88 a 1994/95, no Estado do Rio Grande do Sul

\begin{tabular}{|c|c|c|c|c|}
\hline Grupos & Locais & Altitude $^{1}$ & Solo $^{2}$ & Clima $^{3}$ \\
\hline \multirow{4}{*}{ Subregião I } & Iraí & $235 \mathrm{~m}$ & Neossolo + Chernossolo & $\mathrm{Cfa}$ \\
\hline & Cruz Alta & $452 \mathrm{~m}$ & Latossolo Vermelho & $\mathrm{Cfa}$ \\
\hline & Santa Maria & $151 \mathrm{~m}$ & Luvissolo & $\mathrm{Cfa}$ \\
\hline & Encruzilhada do Sul & $432 \mathrm{~m}$ & Neossolo & $\mathrm{Cfb}$ \\
\hline \multirow{2}{*}{ Subregião II } & Frederico Westphalen & $566 \mathrm{~m}$ & Neossolo + Chernossolo & $\mathrm{Cfa}$ \\
\hline & Erechim & $783 \mathrm{~m}$ & Neossolo + Chernossolo & $\mathrm{Cfa}$ \\
\hline \multirow{3}{*}{ Subregião III } & Pelotas - EEC ${ }^{4}$ & $168 \mathrm{~m}$ & Argissolo & $\mathrm{Cfa}$ \\
\hline & Capão do Leão & $21 \mathrm{~m}$ & Planossolo & $\mathrm{Cfa}$ \\
\hline & Maquiné & $12 \mathrm{~m}$ & Neossolo + Chernossolo & $\mathrm{Cfa}$ \\
\hline \multirow{3}{*}{ Subregião IV } & Sarandi & $503 \mathrm{~m}$ & Latossolo & $\mathrm{Cfa}$ \\
\hline & Estrela & $43 \mathrm{~m}$ & Neossolo + Chernossolo & $\mathrm{Cfa}$ \\
\hline & Veranópolis & $705 \mathrm{~m}$ & Latossolo Bruno & $\mathrm{Cfb}$ \\
\hline Subregião V & Passo Fundo & $678 \mathrm{~m}$ & Latossolo Vermelho & $\mathrm{Cfa}$ \\
\hline Subregião VI & Canguçu & $386 \mathrm{~m}$ & Chernossolo + Neossolo & $\mathrm{Cfb}$ \\
\hline
\end{tabular}

Rev. Ceres, Viçosa, v. 59, n.2, p. 213-224, mar/abr, 2012 
ção considerável nesses oito anos, com extremos em 06/ 09 e 30/11. Por outro lado, Piana et al. (2007), analisando o subconjunto de 33 ambientes em que foram registrados dados do estande dos 89 ambientes considerados nesta pesquisa, encontraram efeito significativo do estande final sobre o rendimento de grãos e variação de 3 a 118 plantas por parcela, embora o estande ideal fosse de 72 plantas.

Observe-se, também, que inferências obtidas de pesquisa como essa estão sujeitas às restrições decorrentes da estrutura do experimento. No presente caso, a circunstância especialmente condicionante é a elevada variação do conjunto de locais entre os anos e sua distribuição na região de cultivo de feijão do RS. Para pesquisas de regionalização, é recomendável a utilização de dados de experimentos conduzidos em locais mais uniformemente distribuídos sobre a região de cultivo.

Por fim, cabe ressaltar que o objetivo desta pesquisa foi regionalização de longo prazo. Os resultados obtidos não se aplicam à regionalização da indicação de cultivares para o próximo período de cultivo. Para esse propósito, as inferências devem restringir-se aos cultivares particulares sob consideração no experimento para determinação do valor de cultivo e uso (VCU), em cada período de avaliação.

\section{CONCLUSÕES}

O desempenho relativo dos genótipos de feijão, na região de cultivo do Estado do Rio Grande do Sul, varia entre ambientes (combinações de locais e anos).

O padrão de heterogeneidade do desempenho relativo dos genótipos de feijão entre os locais dessa região não é consistente entre anos, o que impossibilita a caracterização de uma regionalização de longo prazo para controle da interação genótipo $\mathrm{x}$ ambiente.

Um problema fundamental para as inferências baseadas em experimentos regionais são as falhas de técnicas experimentais, particularmente a falta de uniformidade dessas técnicas entre ambientes.

Metodologias baseadas na máxima verossimilhança possibilitam inferências sobre os componentes de variância da interação genótipo x ambiente, por procedimentos que levam em conta a heterogeneidade de variância do erro experimental e a estrutura de dados incompleta, que são usuais em experimentos regionais.

\section{AGRADECIMENTOS}

À Capes e ao CNPq, pela concessão de bolsas de pesquisa. A todas as instituições que colaboraram com a execução do Ensaio Estadual do Feijão no período de 1987/ 88 a 1994/95.

\section{REFERÊNCIAS}

Abou-El-Fittouh H, Rawlings JO \& Miller PA (1969) Classification of environments to control genotypes by environment interaction to cotton. Crop Science, 9:135-140.

Bartlett MS (1937) Some examples of statistics methods of research in agriculture and applied biology. Journal of Royal Statistics Society, 4:137-183.

Byth DE, Eisemann RL \& De Lacy HI (1976) Two-way pattern analysis of a large data set to evaluate genotype adaptation. Heredity, 37:215-230.

Campbell LG \& Lafaver HN (1977) Cultivar x environment interaction in soft red winter yield tests. Crop Science, 17:607-608.

Carbonell SAM \& Pompeu AS (1997) Estratificação de ambientes em experimentos de feijoeiro no Estado de São Paulo. Bragantia, 56:207-218.

Cochran WG (1954) The combination of estimate from different experiment. Biometrics, 10:101-129.

CONAB - Companhia Nacional de Abastecimento (2011) Ministério da Agricultura, Pecuária e Abastecimento Safras. Séries históricas relativas às safras 1976/77 a 2009/2010. Disponível em: <http://www.conab.gov.br/conteudos.php? $\mathrm{a}=1252 \&$ ordem $=$ produto\&Pagina_objcmsconteudos $=2 \#$ A_objcmsconteudos $>$. Acessado em: 24 de outubro de 2011.

Cordeiro CMT \& Silva JGC da (1980) Estudo do zoneamento da região Centro-Sul do Brasil para a cultura do milho. Pesquisa Agropecuária Brasileira, 15:191-205.

EMBRAPA (2006) Sistema Brasileiro de Classificação de Solos. $2^{\text {a }}$ ed. Rio de Janeiro, Embrapa Solos. 306p.

Garbuglio DD, Gerage AC, Araújo PM de, Fonseca Junior N da S \& Shioga PS (2007) Análise de fatores e regressão bissegmentada em estudos de estratificação ambiental e adaptabilidade em milho. Pesquisa Agropecuária Brasileira, 42:183-191.

IPAGRO - Instituto de Pesquisas Agronômicas (1986) Recomendações para a cultura do feijão. Porto Alegre, Secretaria da Agricultura. $46 \mathrm{p}$.

Liang GHL, Heyne EG \& Walter TL (1966) Estimates of variety $\mathrm{x}$ environmental interactions in yield tests of three small grains and their significance on the breeding programs. Crop Science, 6:135-139.

Littell RC, Milliken GA, Stroup WW, Wolfinger RD, Schabenberber O \& Oliver PD (2006) SAS System for Mixed Models. $2^{\mathrm{a}}$ ed. Cary, SAS Institute Inc. $831 \mathrm{p}$.

Mendonça O, Carpentieri-Pípolo V, Garbuglio DD \& Fonseca Junior N da S (2007) Análise de fatores e estratificação ambiental na avaliação da adaptabilidade e estabilidade em soja. Pesquisa Agropecuária Brasileira, 42:1567-1575.

Mota FS (1951) Estudos do clima do Estado do Rio Grande do Sul segundo o sistema de W. Koeppen. Revista Brasileira de Geografia, 13:107-116.

Mungomery VE, Shorter R \& Byth DE (1974) Genotype x environment interactions and environmental adaptation. I. Pattern analysis - Application to soya bean populations. Australian Journal of Agricultural Research, 25:59-72.

Murakami DM \& Cruz CD (2004) Proposal of methodologies for environment stratification and analysis of genotype adaptability. Crop Breeding and Applied Biotechnology, 4:7-11.

Oliveira GV de, Carneiro PC de S, Dias LA dos S, Carneiro JE de S \& Cruz CD (2005) Factor analysis in the environment stratification for the evaluation of common bean cultivars. Crop Breeding and Applied Biotechnology, 5:166-173. 
Patterson HD \& Thompson WA (1971) Recovery of inter-block information when block sizes are unequal. Biometrika, 58:545554.

Piana CF de B, Silva JGC da \& Antunes IF (2007) Ajuste do rendimento para a variação do estande em experimentos de melhoramento genético do feijão. Pesquisa Agropecuária Brasileira, 42:1687-1696.

Porto RP \& Cortazzi AM (1985) Carta de solos do Rio Grande do Sul. Porto Alegre, Secretaria da Agricultura. (Mapa)

SAS Institute INC (2008) SAS OnlineDoc® 9.1.3. Cary, SAS Institute Inc. Disponível em: <http://support.sas.com/onlinedoc/ 913/docMainpage.jsp>. Acessado em: 17 de outubro de 2010.

Satterthwaite FC (1946) An approximate distribution of estimates of variance components. Biometrics, 2:110-114.

Searle SR, Casella G \& Mcculloch CE (1992) Variance components. New York, John Wiley. 531p.

Silva EC da (1982) Classificações ambientais para controlar a interação genótipo $\mathrm{x}$ ambiente com aplicação à cultura do trigo no Rio Grande do Sul. Pesquisa Agropecuária Brasileira, 17:757-766.
Silva JGC da (1997) Problems and solutions in the statistical analysis of wide-range experiments: heterogeneity of variance. Biometric Bulletin, 14:15.

Sneath PHA \& Sokal RR (1973) Principles of numerical taxonomy. San Francisco, Freeman. 573p.

Sokal RR \& Michener CD (1958) A statistical method for evaluating systematic relationships. The University of Kansas Science Bulletin, 38:1409-1438.

Thompson WA (1962) The problem of negative estimates of variance components. Annals of Mathematical Statistics, 33:273-289.

Wikipédia (2010) A enciclopédia livre. Rio Grande do Sul. Mesorregiões, microrregiões e municípios. Disponível em: <http://pt.wikipedia.org/wiki/Rio_grande_do_sul>. Acessado em: 17 de outubro de 2010. 